\title{
Liquid Chromatographic Resolution of Tocainide and Its Analogues on a Doubly Tethered Chiral Stationary Phase Based on (+)-(18-Crown-6)-2,3,11,12-tetracarboxylic Acid ${ }^{\dagger}$
}

\author{
Hee Jin Kim, Hee Jung Choi, and Myung Ho Hyun* \\ Department of Chemistry and Chemistry Institute for Functional Materials, Pusan National University, \\ Pusan 609-735, Korea.*E-mail:mhhyun@pusan.ac.kr \\ Received October 21, 2009, Accepted December 28, 2009
}

\begin{abstract}
A doubly tethered chiral stationary phase (CSP) based on (+)-(18-crown-6)-2,3,11,12-tetracarboxylic acid were applied to the liquid chromatographic resolution of racemic tocainide, an antiarrhythmic agent, and its analogues. The chiral recognition efficiency of the doubly tethered CSP for tocainide and its analogues was generally greater than that of the corresponding singly tethered CSP especially in terms of the resolution $\left(\mathrm{R}_{\mathrm{S}}\right)$. The resolution of tocainide and its analogues on the doubly tethered CSP were dependent on the content and the type of the organic and acidic modifiers in aqueous mobile phase and the column temperature. Especially, the retention behaviors of analytes on the doubly tethered CSP with the variation of the content of organic modifier in aqueous mobile phase were opposite to those on the corresponding singly tethered CSP and these opposite retention behaviors were rationalized by the lipophilicity differences of the two CSPs.
\end{abstract}

Key Words: Chiral stationary phase, Enantiomer separation, Liquid chromatography, (+)-(18-crown-6)-2,3, 11,12-tetracarboxylic acid, Tocainide

\section{Introduction}

Tocainide, 2-amino- $N$-(2,6-dimethylphenyl)-propanamide, containing a chiral center is a well known antiarrhythmic agent. ${ }^{1}$ The $(R)$-enantiomer of tocainide was reported to be three times more potent than the $(S)$-enantiomer. ${ }^{2}$ Consequently, the determination of the enantiomeric composition of tocainide is quite important. Among various chiral analysis methods for the determination of the enantiomeric composition of chiral drugs, liquid chromatographic separation of enantiomers on chiral stationary phases (CSPs) has been known to be very useful. ${ }^{3}$ Previously, several liquid chromatographic CSPs were utilized for the resolution of tocainide. For example, $\alpha_{1}$-acid glycoprotein silica (AGP), ${ }^{4}$ cellulase (CBH I) silica ${ }^{5}$ and modified diallyl-tartaramide crosslinked in network and immobilized on silica ${ }^{6}$ have been used in the liquid chromatographic resolution of tocainide. Tocainide has one primary amino group. Consequently tocainide can be resolved on crown ether-based CSPs, which have been known to be very effective for the resolution of racemic compounds containing a primary amino group. ${ }^{7}$

In our laboratory, we have developed a CSP (CSP 1, Figure 1) by bonding (+)-(18-crown-6)-2,3,11,12-tetracarboxylic acid to aminopropylsilica gel. CSP 1 was quite effective for the resolution of $\alpha-,{ }^{8} \beta-,{ }^{9}$ and $\gamma$-amino acids, ${ }^{10}$ racemic primary amines, ${ }^{11}$ amino alcohols, ${ }^{11}$ racemic fluoroquinolone antibacterials, ${ }^{12}$ $\alpha$-amino ketones ${ }^{13}$ and di- and tri-peptides. ${ }^{14}$ CSP 1 was also applied to the resolution of tocainide and its analogues. ${ }^{15}$ The separation of racemic primary amino compounds on CSP 1 has been usually performed under highly acidic condition with an aqueous mobile phase containing sulfuric acid. In this instance, CSP 1 was not ensured to be stable enough for the use of long

${ }^{\dagger}$ This paper is dedicated to Professor Sunggak Kim on the occasion of his honorable retirement. time. To improve the stability of CSP 1 , we have developed a doubly tethered CSP (CSP 2, Figure 1) by attaching the second tethering group to silica gel through a carbon atom of the first tethering group of CSP $1 .{ }^{16} \mathrm{CSP} 2$ was found to be more stable than CSP 1 as expected and, in addition, CSP 2 was found to be generally superior to CSP $\mathbf{1}$ in the resolution of various racemic $\alpha$ - and $\beta$-amino acids, primary amino alcohols and primary amines. ${ }^{16,17}$ However, tocainide and its analogues have not been resolved on CSP 2.

In this study, we wish to elucidate that CSP 2 is also effective for the resolution of tocainide and its analogues. By comparing the chromatographic resolution results on CSP 2 with those on CSP 1, we expect that the characteristics of CSP 2 in the resolution of tocainide and its analogues are explored.

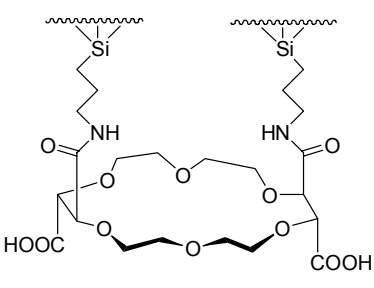

CSP 1

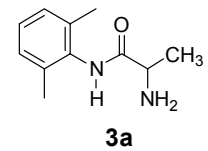

$3 a$

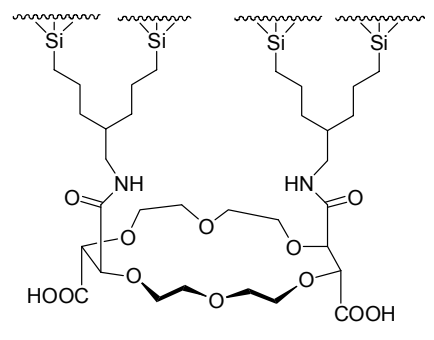

CSP 2

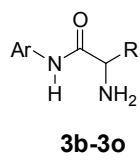

Figure 1. Structures of CSP 1, CSP 2, tocainide (3a) and tocainide analogues $(\mathbf{3 b}-\mathbf{3 o})$. 


\section{Experimental}

Chromatography was performed with an HPLC system consisting of a Waters model 510 HPLC Pump, a Rheodyne model $7725 \mathrm{i}$ injector with a $20 \mu \mathrm{L}$ sample loop, a Waters 486 Absorbance Detector and a YoungLin Autochro Data Module (Software: YoungLin Autochro-2000). The temperature of the chiral column was controlled by using a JEIO TECH VTRC-620 Circulator (Seoul, Korea). Chiral column $(150 \mathrm{~mm} \times 4.6 \mathrm{~mm}$ I.D. $)$ packed with CSP 2 was available from prior study. ${ }^{16}$ Each of racemic and optically active tocainide (3a) and its analogues (3b-3o) shown in Figure 1, which were prepared from the corresponding racemic and optically active $\alpha$-amino acids in this laboratory, were available from previous study. ${ }^{15}$ Each of racemic and optically active tocainide (3a) and its analogues (3b-3o) was dissolved in water (usually $2.5 \mathrm{mg} / \mathrm{mL}$ ) and then used for the resolution on CSP 2. The usual injection volume was $0.1 \mu \mathrm{L}$.

\section{Results and Discussion}

Tocainide (3a) and its analogues (3b-3o) were resolved on CSP 2 with the use of $80 \%$ methanol in water containing $10 \mathrm{mM}$ sulfuric acid as a mobile phase and the chromatographic resolution results were summarized and compared to those on CSP 1 in Table 1. The elution orders shown in Table 1 are identical on the two CSPs and consequently the identical chiral recognition mechanism is expected to operate on the two CSPs. In the resolution of tocainide, CSP 2 was found to be much better than CSP 1 in terms of the separation factor $(\alpha)$ and the resolution $\left(R_{S}\right)$. While baseline separation of the two enantiomers of tocainide is not observed on CSP $1\left(\mathrm{R}_{S}=0.92\right)$, clean baseline separation is observed on CSP $2\left(R_{S}=3.03\right)$. Consequently CSP 2 can be practically applied to the determination of the enantiomeric puri- (a)

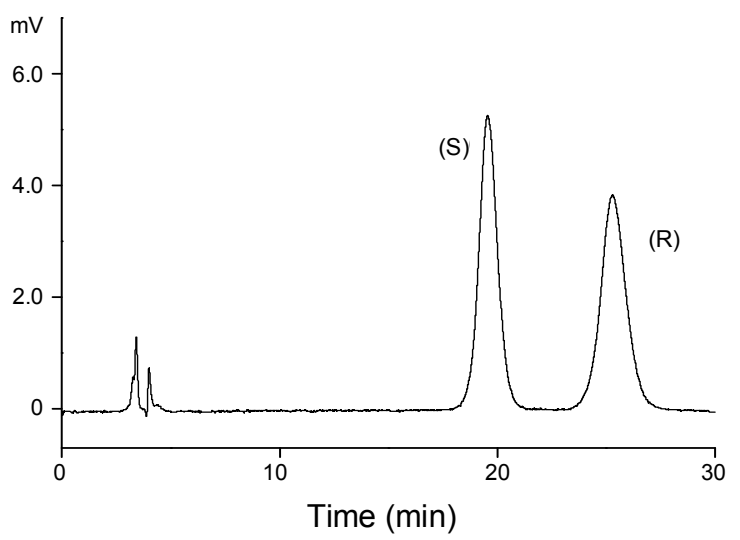

(b)

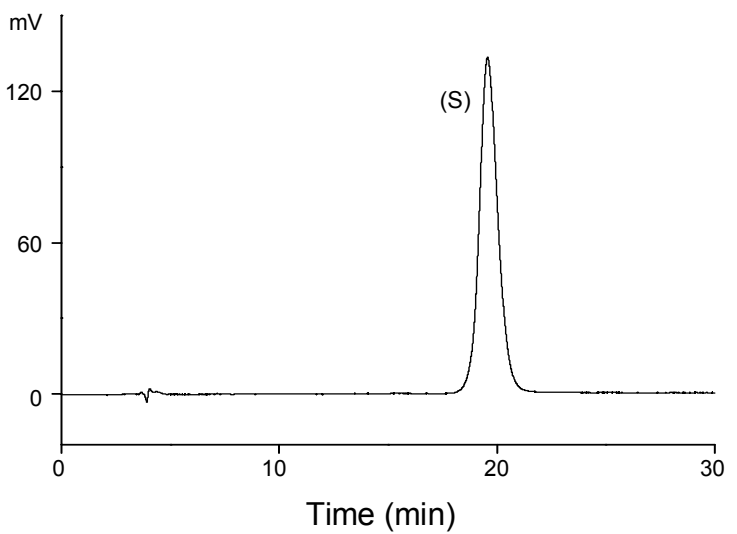

Figure 2. (a) Chromatogram for the resolution of racemic tocainide (3a) on CSP 2. (b) Chromatogram for the resolution of $(S)$-tocainide prepared from $(S)$-alanine on CSP 2 . Mobile phase: $80 \%$ methanol in water containing sulfuric acid $(10 \mathrm{mM})$. Flow rate: $0.5 \mathrm{~mL} / \mathrm{min}$. Detection: $210 \mathrm{~nm} \mathrm{UV}$. Column temperature: $20^{\circ} \mathrm{C}$.

Table 1. Comparison of the resolution of tocainide (3a) and its analogues (3b-3o) on CSP $\mathbf{1}$ and CSP $\mathbf{2}^{a}$

\begin{tabular}{|c|c|c|c|c|c|c|c|c|}
\hline & \multicolumn{2}{|c|}{ analytes } & \multicolumn{3}{|c|}{ CSP 1} & \multicolumn{3}{|c|}{ CSP 2} \\
\hline & $\mathrm{R}$ & $\mathrm{Ar}$ & $k_{1}$ & $\alpha$ & $\mathrm{R}_{\mathrm{S}}$ & $k_{1}$ & $\alpha$ & $\mathrm{R}_{\mathrm{S}}$ \\
\hline $\mathbf{3 a}$ & Methyl & 2,6-Dimethylphenyl & $1.05(\mathrm{~S})$ & 1.17 & 0.92 & $5.46(\mathrm{~S})$ & 1.35 & 3.03 \\
\hline $\mathbf{3 b}$ & Methyl & Phenyl & $1.82(\mathrm{~S})$ & 1.73 & 2.52 & $8.02(\mathrm{~S})$ & 1.63 & 4.52 \\
\hline $3 c$ & Methyl & Benzyl & $1.38(\mathrm{~S})$ & 1.44 & 2.10 & $6.87(\mathrm{~S})$ & 1.39 & 3.10 \\
\hline 3d & Isopropyl & 2,6-Dimethylphenyl & 0.10 & 1.00 & & $1.03(\mathrm{~S})^{b}$ & 1.24 & 1.34 \\
\hline $3 e$ & Isopropyl & Phenyl & $0.34(\mathrm{~S})$ & 2.10 & 2.56 & $1.88(\mathrm{~S})$ & 1.81 & 5.33 \\
\hline $3 f$ & Isopropyl & Benzyl & $0.30(\mathrm{~S})$ & 1.42 & 1.00 & $1.85(\mathrm{~S})$ & 1.18 & 1.55 \\
\hline $3 g$ & Isobutyl & 2,6-Dimethylphenyl & $0.25(\mathrm{~S})$ & 1.17 & 0.25 & $1.33(\mathrm{~S})$ & 1.29 & 2.09 \\
\hline $3 \mathbf{h}$ & Isobutyl & Phenyl & $1.35(\mathrm{~S})$ & 5.00 & 4.00 & $7.34(\mathrm{~S})$ & 3.78 & 10.61 \\
\hline $3 \mathbf{i}$ & Isobutyl & Benzyl & $1.07(\mathrm{~S})$ & 2.39 & 5.50 & $6.11(\mathrm{~S})$ & 1.99 & 6.62 \\
\hline $3 \mathbf{j}$ & Benzyl & 2,6-Dimethylphenyl & 0.58 & 1.00 & & $3.06(\mathrm{~S})$ & 1.15 & 1.13 \\
\hline $3 \mathbf{k}$ & Benzyl & Phenyl & $2.29(\mathrm{~S})$ & 3.72 & 5.33 & $13.05(\mathrm{~S})$ & 2.37 & 6.83 \\
\hline 31 & Benzyl & Benzyl & $2.08(\mathrm{~S})$ & 2.19 & 3.29 & $12.64(\mathrm{~S})$ & 1.60 & 4.84 \\
\hline $3 \mathrm{~m}$ & Phenyl & 2,6-Dimethylphenyl & $1.49(\mathrm{~S})$ & 2.05 & 3.52 & $6.85(\mathrm{~S})$ & 1.88 & 5.44 \\
\hline $3 n$ & Phenyl & Phenyl & $1.55(\mathrm{~S})$ & 3.50 & 5.50 & $7.02(\mathrm{~S})$ & 2.75 & 9.69 \\
\hline 30 & Phenyl & Benzyl & $1.60(\mathrm{~S})$ & 2.58 & 4.89 & $7.91(\mathrm{~S})$ & 2.16 & 8.09 \\
\hline
\end{tabular}

${ }^{a}$ Chromatographic data for CSP 1 are quoted from Ref. 15 . Mobile phase: $80 \%$ methanol in water + sulfuric acid (10 mM). Flow rate: $0.5 \mathrm{~mL} / \mathrm{min}$. Detection: $210 \mathrm{~nm}$ UV. Temperature: $20^{\circ} \mathrm{C} . k_{1}$ : Retention factor of the first eluted enantiomer. In the parenthesis, the absolute configuration of the first eluted enantiomer is presented. $\alpha$ : Separation factor. Rs: Resolution. ${ }^{b}$ For the resolution of 3d on CSP 2, 30\% methanol in water containing $10 \mathrm{mM}$ sulfuric acid and $1 \mathrm{mM}$ ammonium acetate was used as a mobile phase. 
Table 2. Resolution of tocainide (3a) and two selected its analogues (3i and $\mathbf{3 k}$ ) on CSP $\mathbf{2}$ with the variation of the content and the type of organic and acidic modifiers in aqueous mobile phase and with the variation of the column temperature ${ }^{a}$

\begin{tabular}{|c|c|c|c|c|c|c|c|c|c|c|}
\hline \multirow{2}{*}{ entry } & \multirow{2}{*}{ mobile phase } & \multicolumn{3}{|c|}{ 3a } & \multicolumn{3}{|c|}{$3 \mathbf{i}$} & \multicolumn{3}{|c|}{$3 \mathbf{k}$} \\
\hline & & $k_{1}$ & $\alpha$ & $\mathrm{R}_{\mathrm{S}}$ & $k_{1}$ & $\alpha$ & $\mathrm{R}_{\mathrm{S}}$ & $k_{1}$ & $\alpha$ & $\mathrm{R}_{\mathrm{S}}$ \\
\hline \multirow{7}{*}{ a } & $30 \% \mathrm{CH}_{3} \mathrm{OH}+\mathrm{H}_{2} \mathrm{SO}_{4}(10 \mathrm{mM}), 20{ }^{\circ} \mathrm{C}$ & $6.10(\mathrm{~S})$ & 1.18 & 1.62 & $14.48(\mathrm{~S})$ & 1.23 & 1.17 & $39.63(\mathrm{~S})$ & 1.32 & 1.61 \\
\hline & $50 \% \mathrm{CH}_{3} \mathrm{OH}+\mathrm{H}_{2} \mathrm{SO}_{4}(10 \mathrm{mM}), 20^{\circ} \mathrm{C}$ & $5.44(\mathrm{~S})$ & 1.26 & 2.15 & $9.58(\mathrm{~S})$ & 1.44 & 3.44 & $22.57(\mathrm{~S})$ & 1.64 & 2.95 \\
\hline & $80 \% \mathrm{CH}_{3} \mathrm{OH}+\mathrm{H}_{2} \mathrm{SO}_{4}(10 \mathrm{mM}), 20^{\circ} \mathrm{C}$ & $5.46(\mathrm{~S})$ & 1.35 & 3.03 & $6.11(\mathrm{~S})$ & 1.99 & 6.62 & $13.05(\mathrm{~S})$ & 2.37 & 6.83 \\
\hline & $30 \% \mathrm{CH}_{3} \mathrm{CH}_{2} \mathrm{OH}+\mathrm{H}_{2} \mathrm{SO}_{4}(10 \mathrm{mM}), 20{ }^{\circ} \mathrm{C}$ & $4.76(\mathrm{~S})$ & 1.23 & 1.79 & $10.93(\mathrm{~S})$ & 1.24 & 1.79 & $29.66(\mathrm{~S})$ & 1.45 & 2.82 \\
\hline & $50 \% \mathrm{CH}_{3} \mathrm{CH}_{2} \mathrm{OH}+\mathrm{H}_{2} \mathrm{SO}_{4}(10 \mathrm{mM}), 20^{\circ} \mathrm{C}$ & $4.76(\mathrm{~S})$ & 1.33 & 2.45 & $8.63(\mathrm{~S})$ & 1.49 & 3.35 & $18.85(\mathrm{~S})$ & 1.88 & 5.41 \\
\hline & $80 \% \mathrm{CH}_{3} \mathrm{CH}_{2} \mathrm{OH}+\mathrm{H}_{2} \mathrm{SO}_{4}(10 \mathrm{mM}), 20^{\circ} \mathrm{C}$ & $5.96(\mathrm{~S})$ & 1.41 & 2.93 & $7.69(\mathrm{~S})$ & 2.03 & 6.33 & $14.56(\mathrm{~S})$ & 2.77 & 9.15 \\
\hline & $80 \% \mathrm{CH}_{3} \mathrm{CN}+\mathrm{H}_{2} \mathrm{SO}_{4}(10 \mathrm{mM}), 20^{\circ} \mathrm{C}$ & 3.36 & 1.00 & & $2.99(\mathrm{~S})$ & 1.68 & 7.54 & $4.85(\mathrm{~S})$ & 2.18 & 10.86 \\
\hline \multirow{8}{*}{$\mathrm{b}$} & $80 \% \mathrm{CH}_{3} \mathrm{OH}+\mathrm{H}_{2} \mathrm{SO}_{4}(1 \mathrm{mM}), 20^{\circ} \mathrm{C}$ & $6.10(\mathrm{~S})$ & 1.18 & 1.62 & $14.48(\mathrm{~S})$ & 1.23 & 1.17 & $39.63(\mathrm{~S})$ & 1.32 & 1.61 \\
\hline & $80 \% \mathrm{CH}_{3} \mathrm{OH}+\mathrm{H}_{2} \mathrm{SO}_{4}(5 \mathrm{mM}), 20^{\circ} \mathrm{C}$ & $5.48(\mathrm{~S})$ & 1.26 & 2.15 & $9.58(\mathrm{~S})$ & 1.44 & 3.44 & $22.57(\mathrm{~S})$ & 1.54 & 2.95 \\
\hline & $80 \% \mathrm{CH}_{3} \mathrm{OH}+\mathrm{H}_{2} \mathrm{SO}_{4}(10 \mathrm{mM}), 20^{\circ} \mathrm{C}$ & $5.46(\mathrm{~S})$ & 1.35 & 3.03 & $6.11(\mathrm{~S})$ & 1.99 & 6.62 & $13.05(\mathrm{~S})$ & 2.37 & 6.83 \\
\hline & $80 \% \mathrm{CH}_{3} \mathrm{CH}_{2} \mathrm{OH}+\mathrm{H}_{2} \mathrm{SO}_{4}(2 \mathrm{mM}), 20^{\circ} \mathrm{C}$ & $14.62(\mathrm{~S})$ & 1.44 & 2.94 & $19.56(\mathrm{~S})$ & 1.87 & 5.08 & $40.61(\mathrm{~S})$ & 2.61 & 7.14 \\
\hline & $80 \% \mathrm{CH}_{3} \mathrm{CH}_{2} \mathrm{OH}+\mathrm{H}_{2} \mathrm{SO}_{4}(5 \mathrm{mM}), 20^{\circ} \mathrm{C}$ & $9.61(\mathrm{~S})$ & 1.43 & 2.97 & $12.25(\mathrm{~S})$ & 1.99 & 5.85 & $24.27(\mathrm{~S})$ & 2.73 & 8.43 \\
\hline & $80 \% \mathrm{CH}_{3} \mathrm{CH}_{2} \mathrm{OH}+\mathrm{H}_{2} \mathrm{SO}_{4}(10 \mathrm{mM}), 20^{\circ} \mathrm{C}$ & $5.96(\mathrm{~S})$ & 1.41 & 2.93 & $7.69(\mathrm{~S})$ & 2.03 & 6.33 & $14.56(\mathrm{~S})$ & 2.77 & 9.15 \\
\hline & $80 \% \mathrm{CH}_{3} \mathrm{CH}_{2} \mathrm{OH}+\mathrm{HClO}_{4}(10 \mathrm{mM}), 20^{\circ} \mathrm{C}$ & $6.40(\mathrm{~S})$ & 1.44 & 3.11 & $8.38(\mathrm{~S})$ & 2.00 & 6.00 & $15.88(\mathrm{~S})$ & 2.66 & 7.91 \\
\hline & $80 \% \mathrm{CH}_{3} \mathrm{CH}_{2} \mathrm{OH}+\mathrm{CF}_{3} \mathrm{CO}_{2} \mathrm{H}(10 \mathrm{mM}), 20^{\circ} \mathrm{C}$ & $5.98(\mathrm{~S})$ & 1.46 & 2.80 & $8.09(\mathrm{~S})$ & 1.94 & 5.19 & $16.19(\mathrm{~S})$ & 2.61 & 7.37 \\
\hline \multirow{3}{*}{$\mathrm{c}$} & $80 \% \mathrm{CH}_{3} \mathrm{CH}_{2} \mathrm{OH}+\mathrm{H}_{2} \mathrm{SO}_{4}(2 \mathrm{mM}), 30^{\circ} \mathrm{C}$ & $4.49(\mathrm{~S})$ & 1.39 & 3.36 & $5.61(\mathrm{~S})$ & 1.94 & 6.80 & $9.92(\mathrm{~S})$ & 2.65 & 9.83 \\
\hline & $80 \% \mathrm{CH}_{3} \mathrm{CH}_{2} \mathrm{OH}+\mathrm{H}_{2} \mathrm{SO}_{4}(2 \mathrm{mM}), 20^{\circ} \mathrm{C}$ & $5.96(\mathrm{~S})$ & 1.41 & 2.93 & $7.69(\mathrm{~S})$ & 2.03 & 6.33 & $14.56(\mathrm{~S})$ & 2.77 & 9.15 \\
\hline & $80 \% \mathrm{CH}_{3} \mathrm{CH}_{2} \mathrm{OH}+\mathrm{H}_{2} \mathrm{SO}_{4}(2 \mathrm{mM}), 10^{\circ} \mathrm{C}$ & $11.29(\mathrm{~S})$ & 1.43 & 2.76 & $14.72(\mathrm{~S})$ & 2.23 & 6.21 & $28.10(\mathrm{~S})$ & 3.08 & 8.48 \\
\hline
\end{tabular}

${ }^{a}$ Flow rate: $0.5 \mathrm{~mL} / \mathrm{min}$. Detection: $210 \mathrm{~nm} \mathrm{UV} . k_{1}$ : Retention factor of the first eluted enantiomer. In the parenthesis, the absolute configuration of the first eluted enantiomer is presented. $\alpha$ : Separation factor. RS: Resolution.

ty of tocainide. The practical usefulness of CSP 2 in the determination of the enantiomeric purity of tocainide is demonstrated by the chromatograms shown in Figure 2. The comparison of the chromatograms for the resolution of racemic tocainide (Figure $2 \mathrm{a})$ and for the resolution of $(S)$-tocainide prepared from $(S)$ alanine (Figure 2b) shows that the enantiomeric purity of the (S)tocainide is more than $99 \%$ ee because the peak corresponding to the $(R)$-tocainide is not observed in Figure $2 \mathrm{~b}$.

In the resolution of tocainide analogues (3b-3o), the separation factors $(\alpha)$ are generally greater on CSP 1 than on CSP 2 except for the resolution of $\mathbf{3 d}, \mathbf{3} \mathbf{g}$ and $\mathbf{3} \mathbf{j}$, but the resolutions $\left(\mathrm{R}_{\mathrm{S}}\right)$ are always greater on CSP $\mathbf{2}$ than on CSP $\mathbf{1}$ as shown in Table 1 . Tocainide analogue $\mathbf{3} \mathbf{j}$ is not resolved at all and $\mathbf{3 g}$ is resolved only slightly on CSP 1 , but these two analytes are resolved quite well on CSP 2. Tocainide analogue $3 \mathrm{~d}$ is not resolved at all on CSP 1 and CSP 2 with the use of $80 \%$ methanol in water containing $10 \mathrm{mM}$ sulfuric acid as a mobile phase, but it is resolved quite well on CSP $\mathbf{2}$ when 30\% methanol in water containing $10 \mathrm{mM}$ sulfuric acid and $1 \mathrm{mM}$ ammonium acetate is used as a mobile phase. Overall, CSP 2 is concluded to be more widely applicable in the resolution of tocainide and its analogues than CSP 1.

The retention times denoted by the retention factors $\left(k_{1}\right)$ are much greater on CSP 2 than on CSP $\mathbf{1}$ as shown in Table 1. By introducing the second tethering group, CSP 2 is expected to become more lipophilic than CSP 1 especially under reverse mobile phase condition and consequently the retention factors $\left(k_{1}\right)$ are expected to be greater on CSP 2 than on CSP 1 as observed in the resolution of $\beta$-amino acids. ${ }^{17}$

In order to characterize the chromatographic behaviors for the resolution of tocainide and its analogues on CSP 2, we three selected analytes ( $\mathbf{3 a}, \mathbf{3 i}$ and $\mathbf{3 k}$ ) and resolved them on CSP $\mathbf{2}$ with the variation of the content and the type of organic and acidic modifiers in aqueous mobile phase and the column temperature. The chromatographic results for the resolution of selected three analytes (3a, $\mathbf{3 i}$ and $\mathbf{3 k}$ ) on CSP $\mathbf{2}$ with the variation of the content and the type of organic and acidic modifiers in aqueous mobile phase and the column temperature are summarized in Table 2. First of all, the effect of the content of organic modifier in aqueous mobile phase on the chromatographic resolution behaviors are quite significant as shown in Table 2 (entry a). Especially the retention behaviors of analytes with the variation of the content of organic modifier in aqueous mobile phase are very interesting. The retention behaviors of the three analytes (3a, 3i and $\mathbf{3 k}$ ) with the variation of the content of organic modifier in aqueous mobile phase are graphically illustrated in Figure 3. When the content of methanol in aqueous mobile phase containing $10 \mathrm{mM}$ sulfuric acid is increased from $30 \%$ to $50 \%$ and then to $80 \%$, the retention factors $\left(k_{1}\right)$ for the resolution of the three analytes (3a, 3i and $\mathbf{3 k}$ ) on CSP 2 decreases continuously (Figure 3b). These retention trends shown in Figure $3 \mathrm{~b}$ are exactly opposite to those shown in Figure $3 \mathrm{a}$ for the resolution of the identical analytes on CSP $1 .{ }^{15}$ Interestingly, the retention factors $\left(k_{1}\right)$ for the resolution of tocainide (3a) on CSP 2 slightly increase as the content of ethanol in aqueous mobile phase is increased while those for the resolution of analytes $\mathbf{3 i}$ and $3 \mathbf{k}$ decrease as shown in Figure $3 \mathrm{c}$. In addition, the decreasing trends of the retention factors $\left(k_{1}\right)$ for the resolution of analytes $3 \mathbf{i}$ and $\mathbf{3 k}$ on CSP $\mathbf{2}$ are less significant with ethanol than with methanol.

The retention behaviors for the resolution of racemic compounds on CSPs based on crown ethers have been proposed to 
(a)

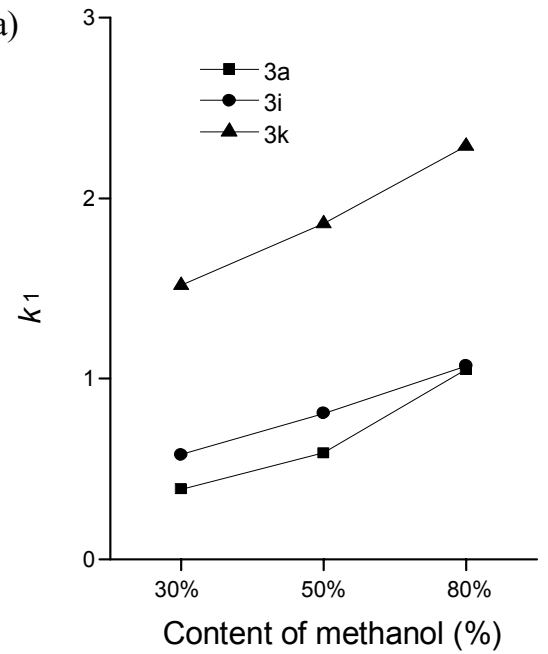

(b)

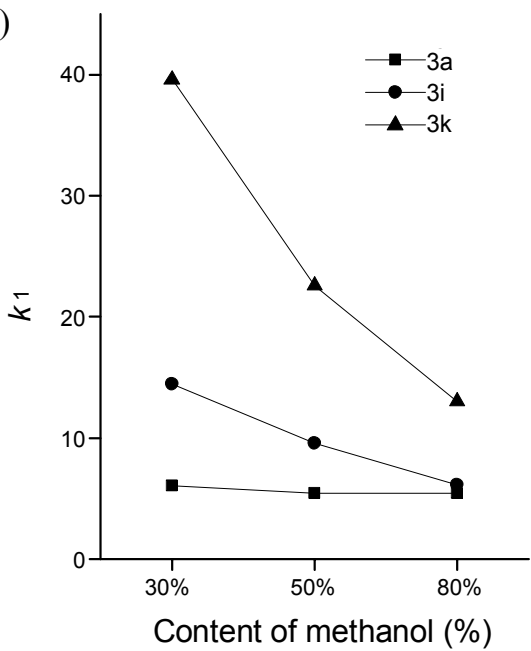

(c)

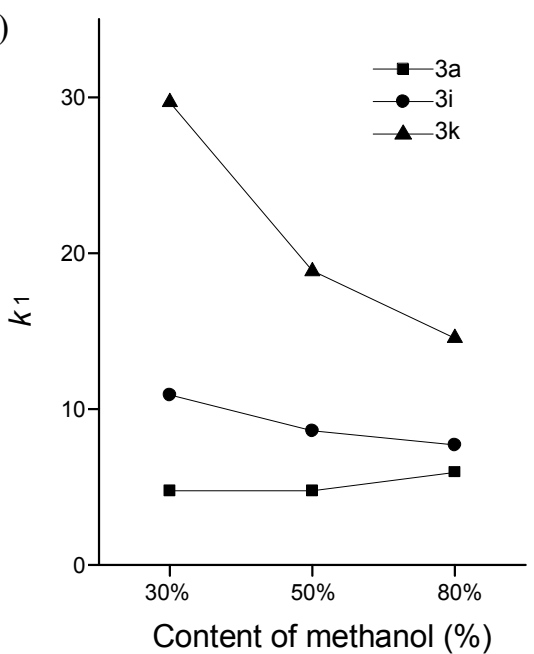

Figure 3. Trends of the retention factors ( $k_{1}$ ) for the resolution of tocainide (3a) and its analogues (3i and $\mathbf{3 k}$ ) on (a) CSP $\mathbf{1}$ and (b) CSP $\mathbf{2}$ with the variation of the content of methanol in aqueous mobile phase containing sulfuric acid $(10 \mathrm{mM})$. (c) Trends of the retention factors $\left(k_{1}\right)$ for the resolution of tocainide (3a) and its analogues (3i and $\mathbf{3 k}$ ) on CSP $\mathbf{2}$ with the variation of the content of ethanol in aqueous mobile phase containing sulfuric acid $(10 \mathrm{mM})$. The retention factor data on CSP 1 are quoted from reference 15 . Flow rate: $0.5 \mathrm{~mL} / \mathrm{min}$. Detection: $210 \mathrm{~nm}$ UV. Temperature: $20{ }^{\circ} \mathrm{C}$.

depend on the balance between the lipophilic interaction of analytes with the CSP and the hydrophilic interaction of analytes with the mobile phase. ${ }^{7}$ When the three analytes (3a, 3i and $\mathbf{3 k}$ ) are resolved on CSP 1, the hydrophilic interaction of analytes with the CSP seems to be predominant over the lipophilic interaction of analytes with the CSP because CSP 1 is relatively less lipophilic. As the content of organic modifier in aqueous mobile phase is increased, the aqueous mobile phase becomes less polar and more hydrophobic and consequently, the hydrophilic interaction of the protonated analytes $\left(\mathrm{R}-\mathrm{NH}_{3}{ }^{+}\right)$with the mobile phase decreases. In this instance, the retention is expected to increase as the content of organic modifier in aqueous mobile phase is increased. Based on this rationale, the increasing trends of the retention factors $\left(k_{1}\right)$ shown in Figure 3 a have been explained. $^{15}$

When a CSP becomes more lipophilic, the lipophilic interaction of analytes with the CSP can be predominant over the hydrophilic interaction of analytes with the mobile phase. As mentioned above, CSP 2 is more lipophilic than CSP 1 because of the second tethering group. In this instance, the lipophilic interaction of analytes with CSP 2 is expected to be predominant over the hydrophilic interaction of analytes with the mobile phase. In this event, as the content of organic modifier in aqueous mobile phase is increased, the polarity of the mobile phase decreases and consequently, the lipophilic interaction of analytes with the CSP decreases and thereby the retention factors $\left(k_{1}\right)$ decrease. The decreasing trends of the retention factors should be more significant with more lipophilic analytes. Analyte 3k might be most lipophilic among three selected analytes because of the two phenyl groups while analyte 3a might be least lipophilic because of the least lipophilic methyl group at the chiral center. As can be seen in Figure 3b, analyte 3k shows the most significant decreasing trends of the retention factors $\left(k_{1}\right)$.

In addition to the lipophilic interaction of analytes with the CSP, complexation of the primary ammonium group $\left(\mathrm{R}_{-} \mathrm{NH}_{3}{ }^{+}\right)$ of analytes inside the cavity of the 18 -crown- 6 ring of the CSP is another important factor for the retention of analytes. ${ }^{18}$ This complexation between the ionic moiety of analytes and the lonepair electrons of the oxygen atoms of the crown ether is more favorable in less polar mobile phase. ${ }^{18}$ When ethanol is used as an organic modifier in aqueous mobile phase, the mobile phase becomes less polar compared to the occasion when methanol in used. The lipophilic interaction of analytes with the CSP should be more significant in more polar mobile phase and consequently the retention of analytes should be greater with methanol than with ethanol as an organic modifier in aqueous mobile phase. However, when the concentration of organic modifier in aqueous mobile phase becomes higher $(80 \%)$, the mobile phase becomes quite less polar and the complexation of the primary ammonium group $\left(\mathrm{R}^{-\mathrm{NH}_{3}}{ }^{+}\right)$of analytes inside the cavity of the 18crown- 6 ring of the CSP becomes important. In this event, the decreasing trends of the retention of analytes is diminished and this phenomena should be more significant with ethanol than with methanol as shown in Figure $3 \mathrm{~b}$ and Figure $3 \mathrm{c}$. In the case of least lipophilic analyte $\mathbf{3} \mathbf{a}$, the retention factor $\left(k_{1}\right)$ even increases when the content of ethanol in aqueous mobile phase is increased to $80 \%$.

The separation factors $(\alpha)$ and the resolutions $\left(R_{S}\right)$ were observed to increase as the content of organic modifier in aqueous mobile phase is increased as shown in Table 2. Complexation of the primary ammonium group $\left(\mathrm{R}_{-} \mathrm{NH}_{3}{ }^{+}\right)$of analytes inside the cavity of the 18-crown-6 ring of the CSP is actually enantioselective. ${ }^{18}$ As mentioned above, this complexation becomes significant as the content of organic modifier in aqueous mobile phase is increased and consequently the chiral recognition efficiency denoted by the separation factors $(\alpha)$ and the resolution $\left(\mathrm{R}_{\mathrm{S}}\right)$ might increase as the content of organic modifier in aqueous mobile phase is increased.

As an organic modifier in aqueous mobile phase, methanol, ethanol or acetonitrile was examined. Even though each of three organic modifiers gave comparable chiral recognition results for the resolution of analytes $\mathbf{3 i}$ and $\mathbf{3 k}$ on CSP $\mathbf{2}$, analyte $\mathbf{3} \mathbf{a}$ was not 


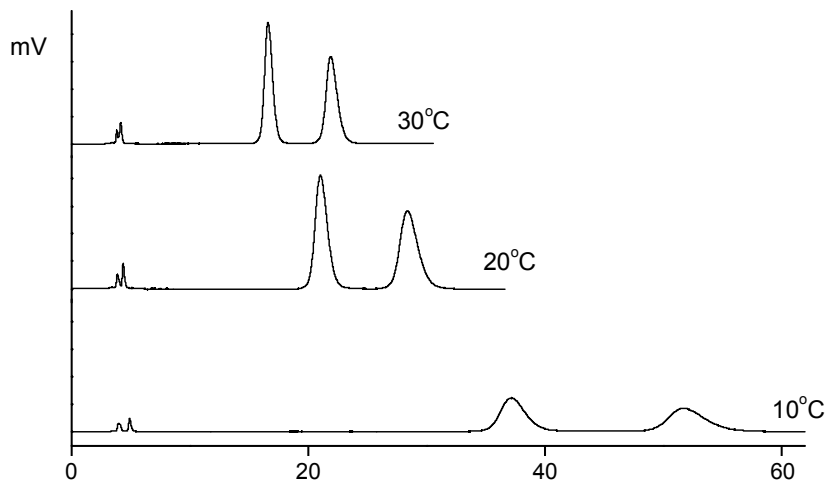

Figure 4. Comparison of the chromatograms for the resolution of tocainide (3a) with the variation of column temperature. Mobile phase: $80 \%$ ethanol in water containing sulfuric acid $(10 \mathrm{mM})$. Flow rate: 0.5 $\mathrm{mL} / \mathrm{min}$. Detection: $210 \mathrm{~nm}$ UV.

resolved at all with acetonitrile as an organic modifier in aqueous mobile phase. The reason is not clear yet.

The acidic modifier added to the mobile phase is used to protonate the primary amino group of the analytes and the resulting primary ammonium ions $\left(\mathrm{R}-\mathrm{NH}_{3}{ }^{+}\right)$have been known to be essential for the chiral recognition through the enantioselective complexation inside the cavity of the crown ether ring of the CSP. ${ }^{7}$ The chromatographic results for the resolution of the three selected analytes $(\mathbf{3 a}, \mathbf{3 i}$ and $\mathbf{3 k}$ ) on CSP $\mathbf{2}$ with the variation of the content and the type of acidic modifier in aqueous mobile phase are summarized in Table 2 (entry $b$ ). The retention factors $\left(k_{1}\right)$ for the resolution of the three selected analytes on CSP 2 decrease continuously as the content of sulfuric acid in aqueous mobile phase is increased and these decreasing trends are exactly consistent with those on CSP $1 .^{15}$ As the content of sulfuric acid in aqueous mobile phase is increased, the ionic strength of mobile phase increases and consequently, the hydration or dissolution of polar-protonated analytes by mobile phase increase and consequently the polar-protonated analytes elute faster. ${ }^{15}$ The separation factors $(\alpha)$ and the resolutions $\left(\mathrm{R}_{\mathrm{S}}\right)$ did not show significant change with the variation of the content of sulfuric acid in aqueous mobile phase. In addition to sulfuric acid, perchloric acid or trifluoroacetic acid was also examined as an acidic modifier in aqueous mobile phase, but notable difference among the three acidic modifiers was not observed.

The resolution of the three selected analytes with the variation of column temperature was performed on CSP 2 and the chromatographic resolution results are summarized in Table 2 (entry c). As the column temperature is reduced from 30 to $20^{\circ} \mathrm{C}$ and then to $10^{\circ} \mathrm{C}$, the retention factors $\left(k_{1}\right)$ and the separation factors $(\alpha)$ increase. The diastereomeric complex formation of the two enantiomers of racemic analytes with the chiral crown ether selector of the CSP is expected to become more favorable at lower temperature and this is more significant with the more stable diastereomeric complex. In this instance, the retention factors $\left(k_{1}\right)$ and the separation factors $(\alpha)$ should increase as the column temperature is decreased. However, the resolutions $\left(\mathrm{R}_{\mathrm{S}}\right)$ were found to decrease as the column temperature is decreased. At lower temperature, the rate of the equilibrium for the formation of the diastereomeric complexes is expected to be slower. In this instance, the chromatographic peaks corresponding to the two enantiomers become broader at lower temperature and consequently, the resolutions decrease as the column temperature is reduced. Figure 4 clearly demonstrates the effect of the column temperature on the resolution of three selected analytes on CSP 2.

In conclusion, CSP 2 prepared previously by introducing the second tethering group into the corresponding singly tethered CSP (CSP 1) was successfully applied to the resolution of tocainide and its analogues. CSP 2 was greater than CSP 1 especially in the resolution of tocainide itself in terms of the separation factor $(\alpha)$ and the resolution $\left(\mathrm{R}_{\mathrm{S}}\right)$. In this event, CSP 2 is practically very useful in the determination of the optical purity of optically active tocainide. The chromatographic behaviors for the resolution of tocainide and its analogues on CSP 2 were dependent on the content and the type of the organic and acidic modifiers in aqueous mobile phase and the column temperature. Especially, the different retention behaviors of analytes between CSP 1 and CSP 2 with the variation of content of organic modifier in aqueous mobile phase were rationalized by the differences in the lipophilicity of the two CSPs.

Acknowledgments. This work was supported by a grant-inaid for the National Core Research Center Program from the Ministry of Education Science \& Technology (R15-2006-02203001-0).

\section{References}

1. Roden, D. M.; Woosley, R. L. New Eng. J. Med. 1986, 315, 41.

2. Jamali, F.; Mehvar, R.; Pasutto, F. M. J. Pharm. Sci. 1989, 78, 695.

3. (a) Ahuja, S. Chiral Separations by Chromatography; American Chemical Society, Washington, D.C., Oxford University Press: Oxford, 2000. (b) Subramanian G., Ed., Chiral Separation techniques: A Practical Approach, Third Completely Revised and Updated Edition; Wiley-VCH: Weinheim, 2007. (c) Hyun, M. H.; Kim, S. N.; Choi, H. J. Bull. Kor. Chem. Soc. 2007, 28, 2531. (d) Choi, H. J.; Kim, Y. K.; Hyun, M. H. Bull. Kor. Chem. Soc. 2008, 29, 1850. (e) Cho, H. S.; Ha, H. J.; Choi, H. J.; Jin, J. S.; Hyun, M. H. Bull. Kor. Chem. Soc. 2008, 29, 2135.

4. Schill, G.; Wainer, I. W.; Barkan, S. A. J. Liq. Chromatogr. 1986, 9, 641 .

5. Marle, I.; Erlandsson, P.; Hansson, L.; Isaksson, R.; Pettersson, C.; Pettersson, G. J. Chromatogr. 1991, 586, 233.

6. Andersson, S.; Allenmark, S.; Moller, P.; Persson, B.; Sanchez, D. J. Chomatogr. A 1996, 741, 23.

7. (a) Hyun, M. H. Bull. Kor. Chem. Soc. 2005, 26, 1153. (b) Choi, H. J.; Hyun, M. H. J. Liq. Chromatogr. Rel. Technol. 2007, 30, 853.

8. Hyun, M. H.; Jin, J. S.; Lee, W. J. Chromatogra. A 1998, 822, 155.

9. Hyun, M. H.; Cho, Y. J.; Jin, J. S. J. Sep. Sci. 2002, 25, 648.

10. Lee, S. J.; Cho, H. S.; Choi, H. J.; Hyun, M. H. J. Chromatogr. A 2008, 1188,318

11. Hyun, M. H.; Jin, J. S.; Koo, H. J.; Lee, W. J. Chromatogr. A 1999 , 837,75 .

12. Hyun, M. H.; Han, S. C.; Koo, H. J.; Lee, W. Chromatographia 2000, 52,473 .

13. Hyun, M. H.; Tan, T.; Cho, Y. J. J. Liq. Chromatogr. Rel. Technol. 2004, 27, 1671.

14. Conrad, U.; Chankvetadze, B.; Scriba, G. K. E. J. Sep. Sci. 2005 , $28,2275$.

15. Hyun, M. H.; Min, H. J.; Cho, Y. J. Bull. J. Kor. Chem. Soc. 2003, 24,911 .

16. Hyun, M. H.; Song, Y.; Cho, Y. J.; Kim, D. H. J. Chromatogr. A 2006, 1108, 208.

17. Hyun, M. H.; Song, Y.; Cho, Y. J.; Choi, H. J. Chirality 2008, 20 , 325 .

18. Hyun, M. H.; Jin, J. S.; Han, S. C.; Cho, Y. J. Microchem. J. 2001, $70,205$. 